\title{
Parametric Macromodeling Based on Amplitude and Frequency Scaled Systems with Guaranteed Passivity
}

\author{
Francesco Ferranti*, Luc Knockaert*, Tom Dhaene*, Giulio Antonini ${ }^{+}$ \\ *Dept. of Information Technology (INTEC), \\ Ghent University-IBBT, Gaston Crommenlaan 8 Bus 201, B-9050, Ghent, Belgium \\ + UAq EMC Laboratory, Dipartimento di Ingegneria Elettrica e dell'Informazione, \\ Università degli Studi dell'Aquila, Via G. Gronchi 18, 67100, L'Aquila, Italy
}

\section{SUMMARY}

We propose a novel parametric macromodeling method for systems described by scattering parameters, which depend on multiple design variables such as geometrical layout or substrate features. It is able to build accurate multivariate macromodels that are stable and passive over the entire design space. Poles and residues are parameterized indirectly. The proposed method is based on an efficient and reliable combination of rational identification, a procedure to find amplitude and frequency scaling

${ }^{*}$ Correspondence to: Francesco Ferranti, Dept. of Information Technology (INTEC),

Ghent University-IBBT, Gaston Crommenlaan 8 Bus 201, B-9050, Ghent, Belgium, e-mail: francesco.ferranti@ugent.be

Contract/grant sponsor: this work is supported by the Research Foundation Flanders (FWO).; contract/grant number:

Received September 2010 
system coefficients, and positive interpolation schemes. Pertinent numerical examples validate the proposed parametric macromodeling technique.

Copyright (c) 2007 John Wiley \& Sons, Ltd.

KEY WORDS: Parametric macromodeling, rational approximation, interpolation, passivity.

\section{Introduction}

Design space exploration, design optimization and sensitivity analysis of electromagnetic (EM) systems are usually performed during a typical design process that consequently requires multiple frequency-domain simulations for different design parameter values (e.g. layout features). Parametric macromodels are suitable to efficiently and accurately perform these design activities, while using multiple EM simulations may often be not feasible due to the high computational cost per simulation. Parametric macromodels approximate the complex behavior of EM systems, which is typically characterized by the frequency (or time) and several design parameters, such as geometrical or substrate features.

Different parametric macromodeling techniques have been developed over the last years. Both poles and residues are parameterized in some approaches [1,2] and this full parameterization allows to model dynamic multivariate data samples. Unfortunately, these techniques are not able to guarantee overall stability and passivity of parametric macromodels. In other formulations, only residues are parameterized [3-5]; this reduces the modeling capability compared with techniques $[1,2]$, but guarantees the stability and passivity of parametric macromodels. Recently, new parametric macromodeling methods in [6-8] parameterize poles and residues and are able to guarantee stability and passivity over the 
entire design space, thereby overcoming some limitations of [1,2] and [3-5]. Unfortunately, these methods can only deal with rational models of the same order and are sensitive to the issues related to the interpolation of state-space matrices [9]. A passivity preserving interpolation of state-space matrices is performed by means of the matrix solution of positivereal and bounded-real lemma, which can be solved using Linear Matrix Inequalities (LMI) or Riccati equation solvers. While the LMI solvers of [10] are significantly faster than classical convex optimization algorithms, the complexity of LMI computations can grow quickly with the number of states $n$. For example, the number of operations required to solve a Riccati equation is $O\left(n^{3}\right)$, while the cost of solving an equivalent LMI is $O\left(n^{6}\right)$ [11]. The method proposed in [8] uses a final optimization procedure with the aim of tuning the accuracy of the parametric macromodel. This optimization step can be computationally expensive and is only described for one parameter in addition to frequency.

This paper proposes a new parametric macromodeling technique for scattering (S) representations, which indirectly parameterizes poles and residues and is able to guarantee stability and passivity over the entire design space. No solution of positive-real and boundedreal lemma is required to ensure overall stability and passivity. The Vector Fitting (VF) technique [12] is initially used to build a set of univariate frequency-dependent macromodels for different combinations of the design variables, as in [3-5]. These initial univariate macromodels are called root macromodels. Stability for each root macromodel is enforced by pole-flipping [12], while passivity is checked and enforced by means of standard techniques (see e.g. [13,14]). Next, amplitude and frequency scaling system coefficients are computed for each root macromodel and properly parameterized by positive interpolation operators [15], [16]. Finally, a parametric macromodel is obtained by a combination of root macromodels and corresponding amplitude 
and frequency scaling coefficients, using positive interpolation schemes that preserve stability and passivity over the complete design space. The proposed technique can deal with root macromodels of different orders, it is able to guarantee overall passivity and stability without solving positive-real and bounded-real lemma and is not sensitive to the issues related to the interpolation of state-space matrices [9]. Some pertinent numerical examples validate the proposed method.

\section{Parametric Macromodeling}

The goal of the present algorithm is to build a parametric representation $\mathbf{R}(s, \boldsymbol{g})$ that accurately models a set of data samples $\left\{(s, \boldsymbol{g})_{k}, \mathbf{H}(s, \boldsymbol{g})_{k}\right\}_{k=1}^{K_{t o t}}$ and guarantees stability and passivity over the entire design space. These data samples depend on the complex frequency $s=j \omega$ and several design variables $\boldsymbol{g}=\left(g^{(m)}\right)_{m=1}^{M}$, such as layout features or substrate parameters. A parametric macromodel in the form

$$
\mathbf{R}(s, \boldsymbol{g})=\mathbf{C}(\boldsymbol{g})(s \mathbf{I}-\mathbf{A}(\boldsymbol{g}))^{-1} \mathbf{B}(\boldsymbol{g})+\mathbf{D}(\boldsymbol{g})
$$

is computed by the proposed parametric macromodeling method. The design space $\mathcal{D}(\boldsymbol{g})$ is considered as the parameter space $\mathcal{P}(s, \boldsymbol{g})$ without frequency. The parameter space $\mathcal{P}(s, \boldsymbol{g})$ contains all parameters $(s, \boldsymbol{g})$. If the parameter space is $(\mathrm{M}+1)$-dimensional, the design space is M-dimensional. Two data grids are used in the modeling process: an estimation grid and a validation grid. The estimation grid is utilized to build the root macromodels. The validation grid is used to validate the modeling capability of the parametric macromodel in a set of points of the design space previously not used for the construction of the root macromodels. 


\subsection{Root Macromodels}

Starting from a set of data samples $\left\{(s, \boldsymbol{g})_{k}, \mathbf{H}(s, \boldsymbol{g})_{k}\right\}_{k=1}^{K_{t o t}}$, a set of frequency-dependent rational macromodels is built for some design space points by means of the VF technique [12]. A pole-flipping scheme is used to enforce stability [12], while passivity assessment and enforcement can be accomplished using the robust standard techniques [13,14]. The result of this initial procedure is a set of rational univariate macromodels, stable and passive, that we call root macromodels. We note that each root macromodel can have a different order. These are the starting points to build a parametric macromodel.

\subsection{Amplitude and Frequency Scaling Coefficients}

Once the root macromodels are available, the next step is gluing them together and building a multivariate representation $\mathbf{R}(s, \boldsymbol{g})$ which models the set of data samples $\left\{(s, \boldsymbol{g})_{k}, \mathbf{H}(s, \boldsymbol{g})_{k}\right\}_{k=1}^{K_{t o t}}$ and preserves stability and passivity over the entire design space. The design space is divided into cells using hyperrectangles (regular grid) [17] or simplices (regular and scattered grid) $[18,19]$. The process of dividing the design space into simplices is called triangulation in 2 $\mathrm{D}$ and tessellation in higher dimensions. A simplex, or N-simplex, is the N-D analogue of a triangle in 2-D and a tetrahedron in $3-\mathrm{D}$, while a hyperrectangle is the $\mathrm{N}-\mathrm{D}$ analogue of a rectangle in 2-D and a rectangular parallelepiped in 3-D. For each data distribution many tessellations can be constructed. The minimal requirement is that the simplices do not overlap, and that there are no holes. Kuhn tessellation [18] can be used for a regular design space grid, this technique splits every hypercube in $\mathbb{R}^{N}$ into N! simplices. Delaunay tessellation [19] can be used for an irregular design space grid, this technique is a space-filling aggregate of simplices and can be performed using standard algorithms [20]. Fig. 1 shows a possible 2-D design space 
divided into cells, in the regular and scattered case, respectively.
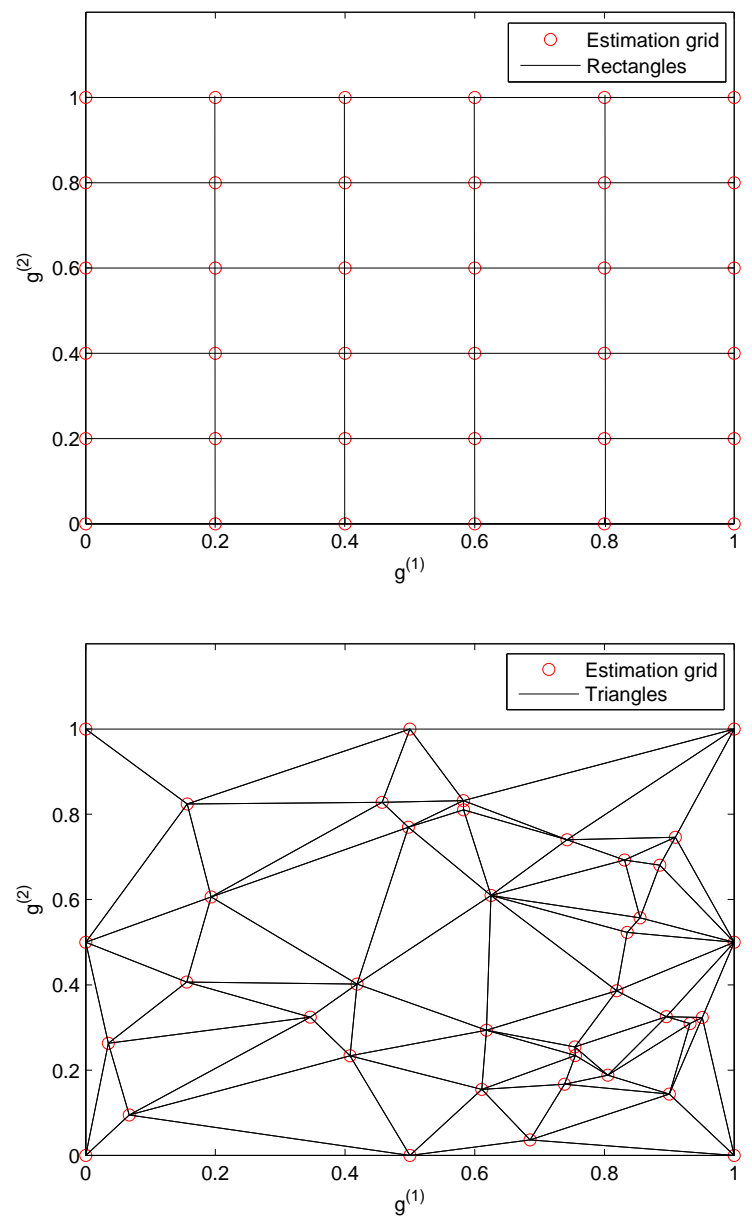

Figure 1. Design space divided into cells: regular (top) and scattered (bottom).

Once the design space is divided into cells, a local parametric macromodel is associated to every cell that is a subdomain of the entire design space. We indicate a cell region of the design space as $\Omega_{i}, i=1, \ldots, P$ and the corresponding vertices as $\boldsymbol{g}_{k}^{\Omega_{i}}, k=1, \ldots, Q$. We note that each vertex corresponds to a root macromodel $\mathbf{R}\left(s, \boldsymbol{g}_{k}^{\Omega_{i}}\right)$. For each cell (a subdomain) of the Copyright (c) 2007 John Wiley \& Sons, Ltd. 
design space, an optimization procedure is used to find the amplitude and frequency scaling system coefficients that make each vertex an accurate approximant of the other cell vertices. For each vertex $\mathbf{R}\left(s, \boldsymbol{g}_{k}^{\Omega_{i}}\right)$, a set of amplitude $\alpha_{k}\left(\boldsymbol{g}_{j}^{\Omega_{i}}\right), j=1, \ldots, Q$ and frequency scaling $\beta_{k}\left(\boldsymbol{g}_{j}^{\Omega_{i}}\right), j=1, \ldots, Q$ real coefficients are found by means of optimization, such that

$$
\begin{gathered}
\alpha_{k}\left(\boldsymbol{g}_{j}^{\Omega_{i}}\right) \mathbf{R}\left(s \beta_{k}\left(\boldsymbol{g}_{j}^{\Omega_{i}}\right), \boldsymbol{g}_{k}^{\Omega_{i}}\right) \simeq \mathbf{R}\left(s, \boldsymbol{g}_{j}^{\Omega_{i}}\right), j \neq k \\
\alpha_{k}\left(\boldsymbol{g}_{j}^{\Omega_{i}}\right)=\beta_{k}\left(\boldsymbol{g}_{j}^{\Omega_{i}}\right)=1, j=k
\end{gathered}
$$

Then, if the response of the system under modeling needs to be computed in a specific design space point $\widehat{\boldsymbol{g}}$, a subdomain that contains $\widehat{\boldsymbol{g}}$ is to be found. For each vertex root macromodel of the found subdomain, the corresponding sets of amplitude and frequency scaling coefficients $\alpha_{k}\left(\boldsymbol{g}_{j}^{\Omega_{i}}\right), \beta_{k}\left(\boldsymbol{g}_{j}^{\Omega_{i}}\right)$ are interpolated in $\widehat{\boldsymbol{g}}$ and a rational model $\widehat{\alpha}_{k} \mathbf{R}\left(s \widehat{\beta}_{k}, \boldsymbol{g}_{k}^{\Omega_{i}}\right)$ is built, where $\widehat{\alpha}_{k}=\alpha_{k}(\widehat{\boldsymbol{g}})$ and $\widehat{\beta}_{k}=\beta_{k}(\widehat{\boldsymbol{g}})$. Finally, the set of modified root macromodels $\widehat{\alpha}_{k} \mathbf{R}\left(s \widehat{\beta}_{k}, \boldsymbol{g}_{k}^{\Omega_{i}}\right), \quad k=1, \ldots, Q$, is interpolated at an input/output level as described in [3-5]. We note that if a generic root macromodel $\mathbf{R}\left(s, \boldsymbol{g}_{k}^{\Omega_{i}}\right)$ has the state-space representation $\{\mathbf{A}, \mathbf{B}, \mathbf{C}, \mathbf{D}\}$, then a corresponding amplitude and frequency scaled version $\widehat{\alpha}_{k} \mathbf{R}\left(s \widehat{\beta}_{k}, \boldsymbol{g}_{k}^{\Omega_{i}}\right)$ has the state-space representation $\{\widetilde{\mathbf{A}}, \widetilde{\mathbf{B}}, \widetilde{\mathbf{C}}, \widetilde{\mathbf{D}}\}$ with

$$
\begin{aligned}
& \widetilde{\mathbf{A}}=\left(\widehat{\beta}_{k}\right)^{-1} \mathbf{A} \\
& \widetilde{\mathbf{B}}=\mathbf{B} \\
& \widetilde{\mathbf{C}}=\widehat{\alpha}_{k}\left(\widehat{\beta}_{k}\right)^{-1} \mathbf{C} \\
& \widetilde{\mathbf{D}}=\widehat{\alpha}_{k} \mathbf{D}
\end{aligned}
$$


2.3. Multivariate Interpolation of Amplitude, Frequency Scaling Coefficients and Root Macromodels

It is known that, while a passive system is also stable, the reverse is not necessarily true [21]. Passivity is crucial when the macromodel is utilized in a circuit simulator (e.g. SPICE [22]) for transient analysis. Passivity refers to the property of systems that cannot generate more energy than they absorb through their electrical ports. When the system is terminated on any arbitrary passive loads, none of them will cause the system to become unstable [23]. The passivity of scattering input-output representations is also called nonexpansivity [24]. A linear network described by scattering matrix $\mathbf{S}(s)$ is passive if [25]:

1. $\mathbf{S}\left(s^{*}\right)=\mathbf{S}^{*}(s)$ for all $s$, where "*" is the complex conjugate operator.

2. $\mathbf{S}(s)$ is analytic in $\Re e(s)>0$.

3. $\mathbf{I}-\mathbf{S}^{\mathrm{T}}\left(s^{*}\right) \mathbf{S}(s) \geq 0 ; \forall s: \Re e(s)>0$.

Condition 3) for nonexpansivity is equivalent to the condition $\|\mathbf{S}(s)\|_{\infty} \leq 1$ ( $\mathbf{H}_{\infty}$ norm) [24], i.e., the largest singular value of $\mathbf{S}(s)$ does not exceed one in the right-half $s$-plane. The interpolated amplitude and frequency scaling real coefficients $\alpha_{k}(\boldsymbol{g}), \beta_{k}(\boldsymbol{g})$ have to satisfy the conditions

$$
\begin{array}{r}
0 \leq \alpha_{k}(\boldsymbol{g}) \leq 1 \\
\beta_{k}(\boldsymbol{g}) \geq 0
\end{array}
$$

to guarantee the passivity of each root macromodel $\alpha_{k}(\boldsymbol{g}) \mathbf{R}\left(s \beta_{k}(\boldsymbol{g}), \boldsymbol{g}_{k}^{\Omega_{i}}\right)$.

Multivariate interpolation based on a class of positive interpolation operators [15], [16] is used to parameterize $\alpha_{k}(\boldsymbol{g}), \beta_{k}(\boldsymbol{g})$. It is able to preserve the passivity of each amplitude and 
frequency scaled root macromodel over the entire design space by guaranteeing the properties (5a)-(5b). The same positive multivariate interpolation schemes are used to interpolate the set of modified root macromodels $\widehat{\alpha}_{k} \mathbf{R}\left(s \widehat{\beta}_{k}, \boldsymbol{g}_{k}^{\Omega_{i}}\right), k=1, \ldots, Q$, at an input/output level, which results in a parametric macromodel, stable and passive over the entire design space.

Multivariate interpolation can be realized by means of tensor product [26] or tessellation [19] methods. Tensor product multivariate interpolation methods require the data points distributed on a fully filled, but not necessarily equidistant, rectangular grid, while tessellationbased multivariate interpolation methods can handle scattered or irregularly distributed data points. Any interpolation scheme based on a class of positive interpolation operators can be used.

In the bivariate case $(s, g)$, each interpolated function $\mathbf{T}(g)$, that can be amplitude, frequency scaling coefficients and root macromodels, can be written as

$$
\mathbf{T}(g)=\sum_{k=1}^{K_{1}} \mathbf{T}_{g_{k}} \ell_{k}(g)
$$

where $K_{1}$ represents the number of root macromodels and each interpolation kernel $\ell_{k}(g)$ is a scalar function satisfying the following constraints

$$
\begin{gathered}
0 \leq \ell_{k}(g) \leq 1, \\
\ell_{k}\left(g_{i}\right)=\delta_{k, i}, \\
\sum_{k=1}^{K_{1}} \ell_{k}(g)=1 .
\end{gathered}
$$


A possible choice is to select $\ell_{k}(g)$ as in piecewise linear interpolation

$$
\begin{gathered}
\frac{g-g_{k-1}}{g_{k}-g_{k-1}}, g \in\left[g_{k-1}, g_{k}\right], k=2, \ldots, K_{1}, \\
\frac{g_{k+1}-g}{g_{k+1}-g_{k}}, g \in\left[g_{k}, g_{k+1}\right], k=1, \ldots, K_{1}-1,
\end{gathered}
$$

0 , otherwise

The bivariate formulation can easily be generalized to the multivariate case by using multivariate interpolation methods that belong to the general class of positive interpolation operators: e.g., the piecewise multilinear and multivariate simplicial methods [17]. We note that the interpolation kernel functions of these methods only depend on the design space grid points and their computation does not require the solution of a linear system to impose an interpolation constraint. In the case of piecewise multilinear interpolation, each interpolated function $\mathbf{T}\left(g^{(1)}, \ldots, g^{(M)}\right)$ can be written as

$$
\begin{aligned}
& \mathbf{T}\left(g^{(1)}, \ldots, g^{(M)}\right)= \\
& =\sum_{k_{1}=1}^{K_{1}} \cdots \sum_{k_{M}=1}^{K_{M}} \mathbf{T}_{\left(g_{k_{1}}^{(1)}, \ldots, g_{k_{M}}^{(M)}\right)} \ell_{k_{1}}\left(g^{(1)}\right) \cdots \ell_{k_{M}}\left(g^{(M)}\right)
\end{aligned}
$$

where each $\ell_{k_{i}}\left(g^{(i)}\right), i=1, \ldots, M$ satisfies constraints $(7)-(9)$ and is selected as in piecewise linear interpolation. Concerning multivariate scattered interpolation, a tessellation-based linear interpolation scheme can be used [5] to build a parametric macromodel. This interpolation method belongs to the class of positive interpolation operators. These positive interpolation schemes have been already used in [3-5], where a parametric macromodel is built by interpolating a set of root macromodels treated as input-output systems, while preserving 
overall stability and passivity. In the proposed new parametric macromodeling technique, a powerful novelty is the use of some interpolated amplitude and frequency scaling system coefficients. It allows to parameterize poles and residues indirectly, hence the modeling capability of the proposed algorithm is increased with respect to [3-5], where the interpolation process were only applied to the root macromodels, and therefore only residues were parameterized.

\subsection{Passivity Preserving Interpolation of Amplitude and Frequency Scaling Coefficients}

In this section, we prove that a passive system $\mathbf{R}(s)$ remains passive if an amplitude scaling coefficient $\alpha$ and a frequency scaling coefficient $\beta$, which satisfy the properties (5a)-(5b), are applied to it. The amplitude scaling coefficient $\alpha$ is a multiplicative factor at the input/output level of the system, while the frequency scaling coefficient $\beta$ is a compression or expansion term for the Laplace variable $s$. It is easy to prove that if $\beta$ satisfies (5b), passivity is preserved, and that if $\alpha$ satisfies (5a), the first two conditions for passivity are preserved. Concerning $\alpha$ and the third passivity condition

$$
\|\alpha \mathbf{R}(\beta s)\|_{\infty}=\alpha\|\mathbf{R}(\beta s)\|_{\infty} \leq \alpha \leq 1
$$

Therefore, if $\alpha$ satisfies (5a), passivity is preserved.

\subsection{Passivity Preserving Interpolation of Root Macromodels}

Once a set of amplitude and frequency scaled root macromodels $\widehat{\alpha}_{k} \mathbf{R}\left(s \widehat{\beta}_{k}, \boldsymbol{g}_{k}^{\Omega_{i}}\right), k=1, \ldots, Q$, which are stable and passive, is built for each cell of the design space, the next step of the proposed method is focused on gluing together these root macromodels by a multivariate 
interpolation scheme to obtain a parametric macromodel $\mathbf{R}(s, \boldsymbol{g})$ with overall stability and passivity. Condition 1) is preserved in (6) and the proposed multivariate extensions, as they are weighted sums with real nonnegative weights of systems respecting this first condition. Condition 2) is preserved in (6) and the proposed multivariate extensions, as they are weighted sums of strictly stable rational macromodels. Condition 3) is equivalent to the condition $\|\mathbf{R}(s)\|_{\infty} \leq 1\left(\mathbf{H}_{\infty}\right.$ norm $)[24]$, therefore in the bivariate case we can write:

$$
\|\mathbf{R}(s, g)\|_{\infty} \leq \sum_{k=1}^{K_{1}}\left\|\mathbf{R}\left(s, g_{k}\right)\right\|_{\infty} \ell_{k}(g) \leq \sum_{k=1}^{K_{1}} \ell_{k}(g)=1
$$

Similar results are obtained for the proposed multivariate cases, so condition 3) is satisfied by construction. We have demonstrated that all three passivity conditions for scattering representations are preserved in the proposed parametric macromodeling algorithm.

\section{Numerical examples}

This section presents two numerical examples to validate the proposed parametric macromodeling approach on application cases. Let us define the absolute error

$$
\begin{aligned}
& \operatorname{Err}(\boldsymbol{g})=\max \left(\left|R_{i, j}\left(s_{k}, \boldsymbol{g}\right)-H_{i, j}\left(s_{k}, \boldsymbol{g}\right)\right|\right) \\
& i=1, \ldots, P_{\text {in }}, j=1, \ldots, P_{\text {out }}, k=1, \ldots, K_{s}
\end{aligned}
$$

where $P_{\text {in }}$ and $P_{\text {out }}$ are the number of inputs and outputs of the system, respectively, and $K_{s}$ is equal to the number of frequency samples. The worst case absolute error over the validation grid is chosen to assess the accuracy and the quality of parametric macromodels 


$$
\begin{gathered}
\boldsymbol{g}_{\max }=\underset{\boldsymbol{g}}{\operatorname{argmax}} \operatorname{Err}(\boldsymbol{g}), \boldsymbol{g} \in \text { validation grid } \\
E r r_{\max }=\operatorname{Err}\left(\boldsymbol{g}_{\max }\right)
\end{gathered}
$$

The number of poles for each root macromodel is selected adaptively in VF by a bottom-up approach, in such a way that the corresponding maximum absolute error is smaller than -60 dB. The proposed macromodeling algorithm is compared with the technique described in $[4,5]$, to confirm its enhanced modeling capability and accuracy.

\subsection{Spiral inductor with variable outer area}

An integrated spiral inductor has been modeled in this example. The structure is shown in Fig. 2. The conductors width is equal to $46 \mu \mathrm{m}$. A bivariate macromodel is built as a function of the outer area $A$ of the spiral inductor in addition to frequency. Their corresponding ranges are shown in Table I.

Table I. Parameters of the spiral inductor.

\begin{tabular}{|l|c|c|}
\hline Parameter & Min & Max \\
\hline Frequency $($ freq $)$ & $10 \mathrm{kHz}$ & $33 \mathrm{GHz}$ \\
Outer area $(A)$ & $460 \mu \mathrm{m}^{2}$ & $930 \mu \mathrm{m}^{2}$ \\
\hline
\end{tabular}

The scattering parameters have been computed by means of an EM solver based on the Partial Element Equivalent Circuit method [27,28] over a validation grid of $250 \times 15$ samples, for frequency and outer area respectively. We have built root macromodels for 8 values of the 


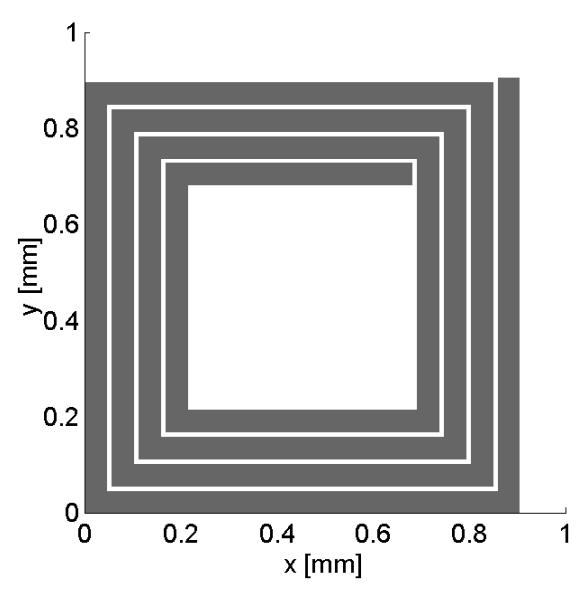

Figure 2. Structure of the spiral inductor.

outer area by means of VF, each with an order chosen by the error-based bottom-up approach described in Section 3. Since the data under modeling are highly dynamic, root macromodels with different orders are required, as shown in Table II. The passivity of each model has been verified by checking the eigenvalues of the corresponding Hamiltonian matrix [24] and enforced, if needed. Finally, a bivariate macromodel is obtained as explained in Section 2, using linear interpolation for the amplitude and frequency scaling coefficients, and root macromodels. Fig. 3 shows the magnitude of the parametric macromodel of $\mathbf{S}_{11}(s, A)$. Figs. 4-5 compare $\mathbf{S}_{11}(s, A)$ and its macromodel for the outer area values $A=\{495,896\} \mu \mathrm{m}^{2}$ that have not been used for the generation of the root macromodels. The worst case absolute error defined in (18) is equal to $2.71 \mathrm{~dB}$ and $-50 \mathrm{~dB}$ for the old $[4,5]$ and new parametric macromodeling techniques, respectively.

As clearly seen, a very good agreement is obtained between the original data and the new proposed parametric macromodeling technique that achieves a better accuracy and modeling 
Table II. Number of poles of root macromodels.

\begin{tabular}{|l|c|}
\hline$A_{1}$ & 8 \\
\hline$A_{2}$ & 10 \\
\hline$A_{3}$ & 10 \\
\hline$A_{4}$ & 10 \\
\hline$A_{5}$ & 10 \\
\hline$A_{6}$ & 12 \\
\hline$A_{7}$ & 14 \\
\hline$A_{8}$ & 14 \\
\hline
\end{tabular}
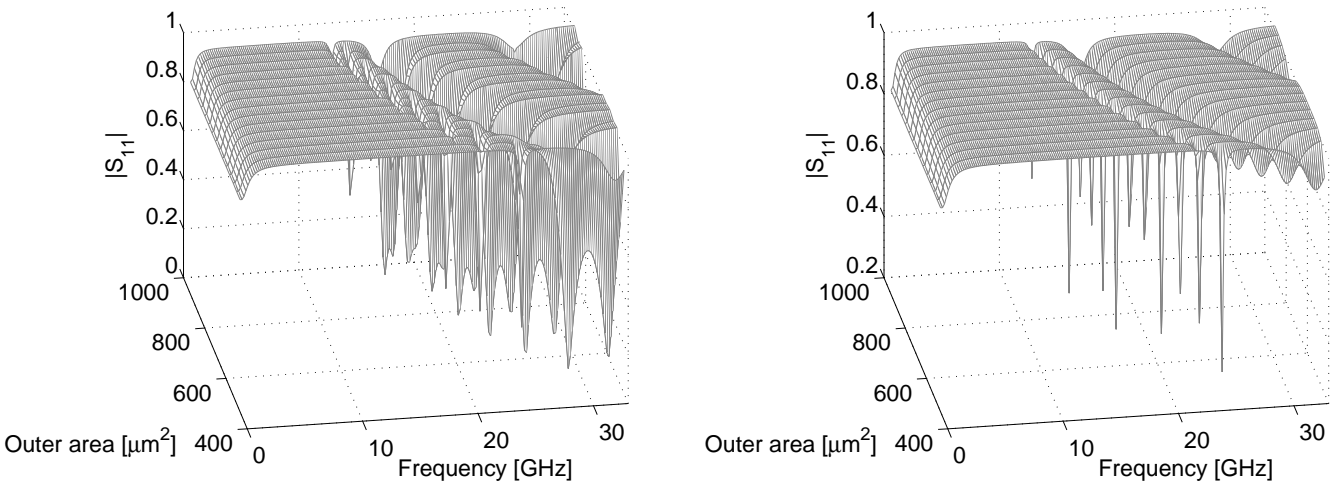

Figure 3. Magnitude of the bivariate macromodel of $\mathbf{S}_{11}(s, A)$ by means of the old technique $[4,5]$ (left) and the new technique (right).

capability with respect to $[4,5]$. The new parametric macromodeling method captures the behavior of the system very accurately, while preserving stability and passivity over the entire design space. 

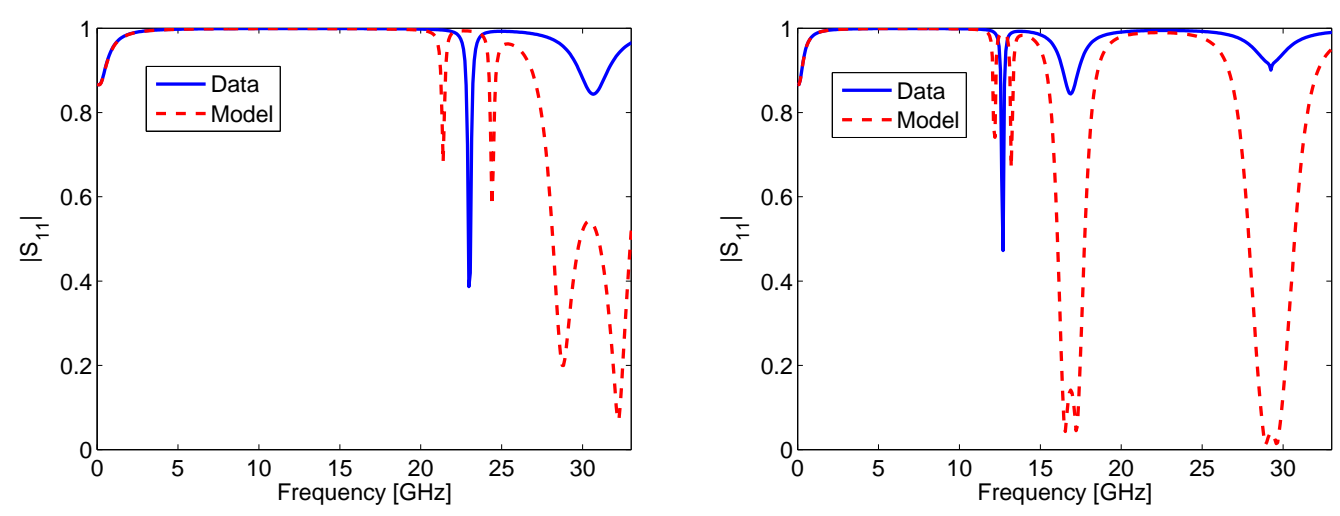

Figure 4. Magnitude of the bivariate macromodel of $\mathbf{S}_{11}(s, A)\left(A=\{476,915\} \mu \mathrm{m}^{2}\right)$ (old technique $[4,5])$.
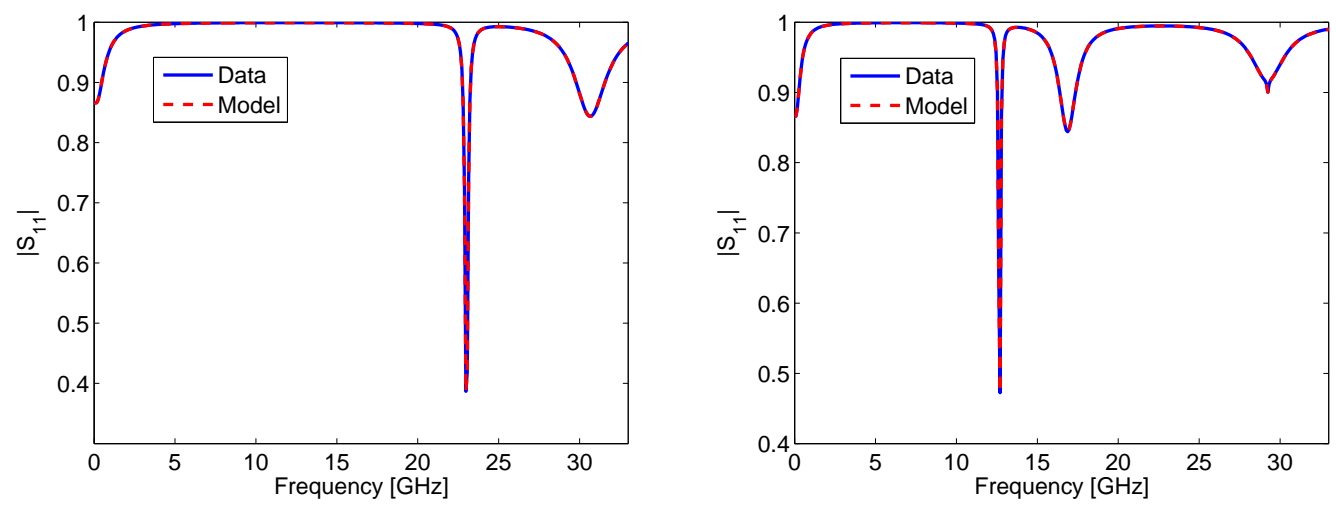

Figure 5. Magnitude of the bivariate macromodel of $\mathbf{S}_{11}(s, A)\left(A=\{476,915\} \mu \mathrm{m}^{2}\right)$ (new proposed technique).

\subsection{Three coupled microstrips with variable length and spacing}

In this second example, three coupled microstrips with frequency-dependent per-unit-length parameters have been modeled. The cross section is shown in Fig. 6 .

The conductors have width $w=100 \mu \mathrm{m}$ and thickness $t=50 \mu \mathrm{m}$. The dielectric is 300 Copyright (c) 2007 John Wiley \& Sons, Ltd. Int. J. Numer. Model. 2007; 01:1-6 Prepared using jnmauth.cls 


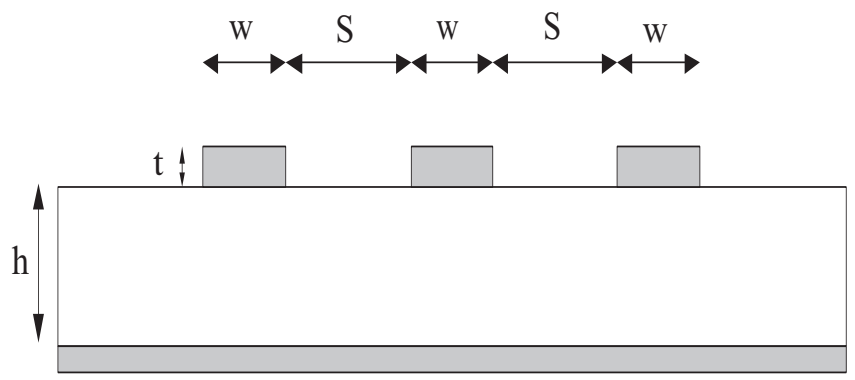

Figure 6. Cross section of the three coupled microstrips.

$\mu \mathrm{m}$ thick and characterized by a dispersive and lossy permittivity which has been modeled by the wideband Debye model [29]. The frequency-dependent per-unit-length parameters have been evaluated using a commercial tool [30]. The length $L$ of the microstrips and the spacing $S$ between the microstrips are considered as parameters in addition to frequency. Their corresponding ranges are shown in Table III.

Table III. Design parameters of the three coupled microstrips structure.

\begin{tabular}{|l|c|c|}
\hline Parameter & Min & Max \\
\hline Frequency (freq) & $100 \mathrm{kHz}$ & $15 \mathrm{GHz}$ \\
Length (L) & $10 \mathrm{~mm}$ & $20 \mathrm{~mm}$ \\
Spacing (S) & $200 \mu \mathrm{m}$ & $400 \mu \mathrm{m}$ \\
\hline
\end{tabular}

The scattering parameters have been computed by means of the exact transmission line theory (TLT) [31] starting from the per-unit-length parameters over a validation grid of $250 \times 13 \times 11$ samples, for frequency, length and spacing, respectively. We have built root macromodels for 7 values of the length and 6 values of the spacing by means of VF, each with Copyright (c) 2007 John Wiley \& Sons, Ltd. Int. J. Numer. Model. 2007; 01:1-6 Prepared using jnmauth.cls 
an order chosen by the error-based bottom-up approach described in Section 3. Similarly to the previous example, root macromodels with different orders are required, as shown in Table IV. Finally, a trivariate macromodel is obtained as explained in Section 2, using multilinear interpolation for the amplitude and frequency scaling coefficients, and root macromodels. Figs. 7-9 show the magnitude of the parametric macromodels of $\mathbf{S}_{11}(s, L, S)$ for the length values $L=\{10,20\} \mathrm{mm}$ and $\mathbf{S}_{14}(s, L, S)$ for the spacing value $S=300 \mu \mathrm{m}$. Figs. 10-13 compare $\mathbf{S}_{11}(s, L, S), \mathbf{S}_{14}(s, L, S)$ and their macromodels for the length values $L=\{10.6,19.4\} \mathrm{mm}$ and the spacing value $S=300 \mu \mathrm{m}$ that have not been used for the generation of the root macromodels. The worst case absolute error defined in (18) is equal to $-18 \mathrm{~dB}$ and $-40 \mathrm{~dB}$ for the old $[4,5]$ and new parametric macromodeling techniques, respectively.

Table IV. Number of poles of root macromodels.

\begin{tabular}{|l|l|l|l|l|l|l|}
\hline & $S_{1}$ & $S_{2}$ & $S_{3}$ & $S_{4}$ & $S_{5}$ & $S_{6}$ \\
\hline$A_{1}$ & 15 & 15 & 15 & 15 & 15 & 15 \\
\hline$A_{2}$ & 18 & 18 & 18 & 18 & 18 & 18 \\
\hline$A_{3}$ & 19 & 19 & 19 & 19 & 19 & 19 \\
\hline$A_{4}$ & 21 & 21 & 21 & 21 & 21 & 21 \\
\hline$A_{5}$ & 23 & 23 & 23 & 23 & 23 & 23 \\
\hline$A_{6}$ & 23 & 23 & 23 & 23 & 23 & 23 \\
\hline$A_{7}$ & 25 & 25 & 25 & 25 & 25 & 25 \\
\hline
\end{tabular}

As in the previous example, the new proposed parametric macromodeling technique shows a better accuracy and modeling capability with respect to $[4,5]$. It is able to accurately describe 

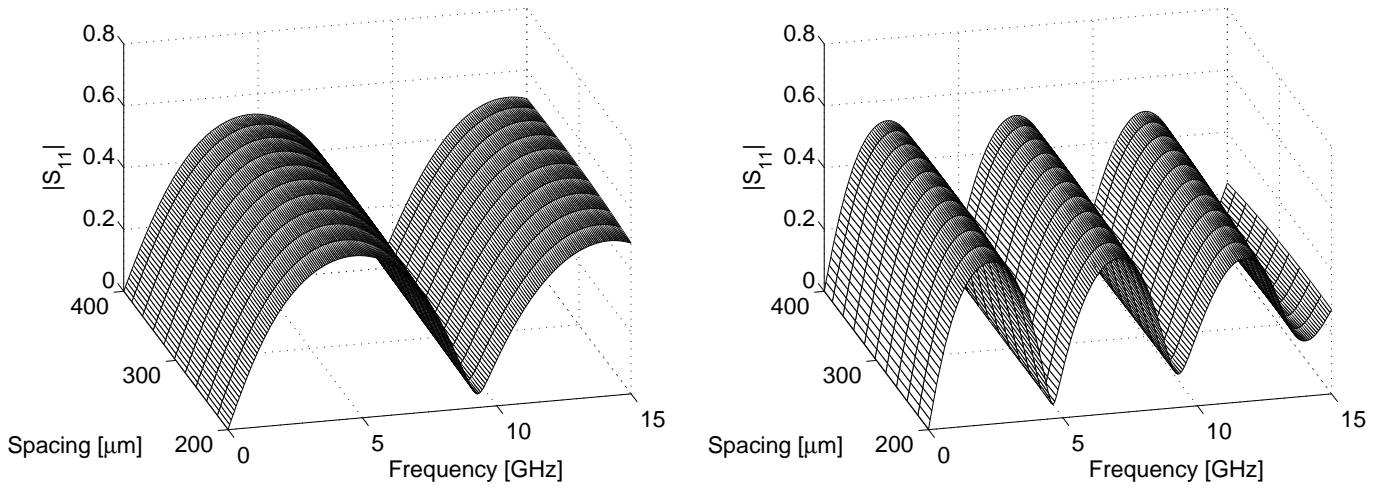

Figure 7. Magnitude of the trivariate macromodel of $\mathbf{S}_{11}(s, L, S)$ for $L=10 \mathrm{~mm}$ (left) and $L=20$ $\mathrm{mm}$ (right) by means of the old technique $[4,5]$.
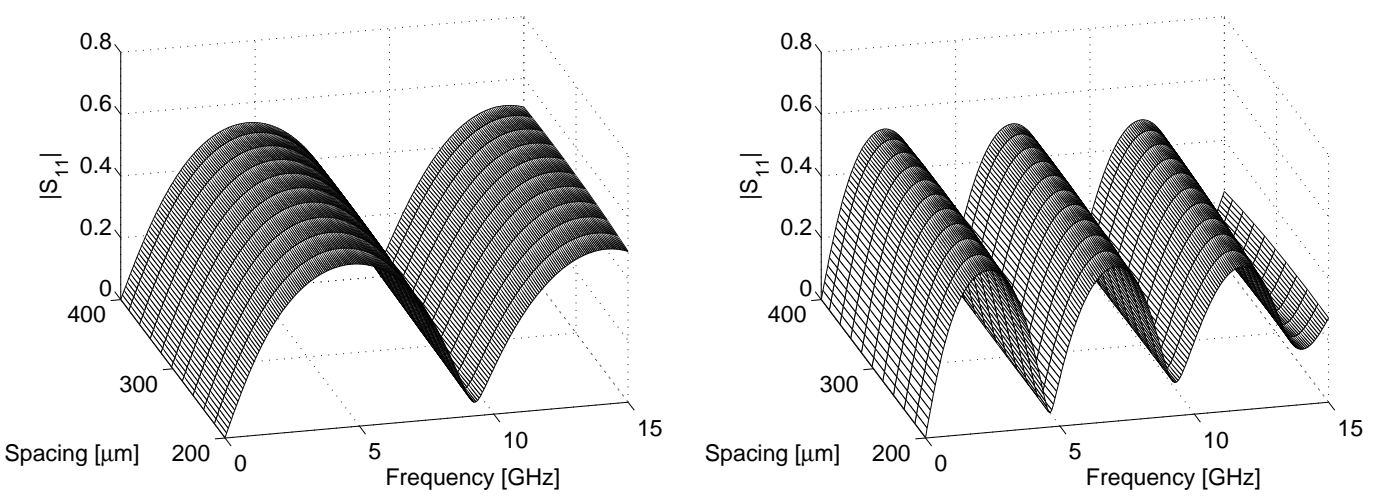

Figure 8. Magnitude of the trivariate macromodel of $\mathbf{S}_{11}(s, L, S)$ for $L=10 \mathrm{~mm}$ (left) and $L=20$ mm (right) by means of the new technique .

the behavior of the system, while stability and passivity are guaranteed over the entire design space. 

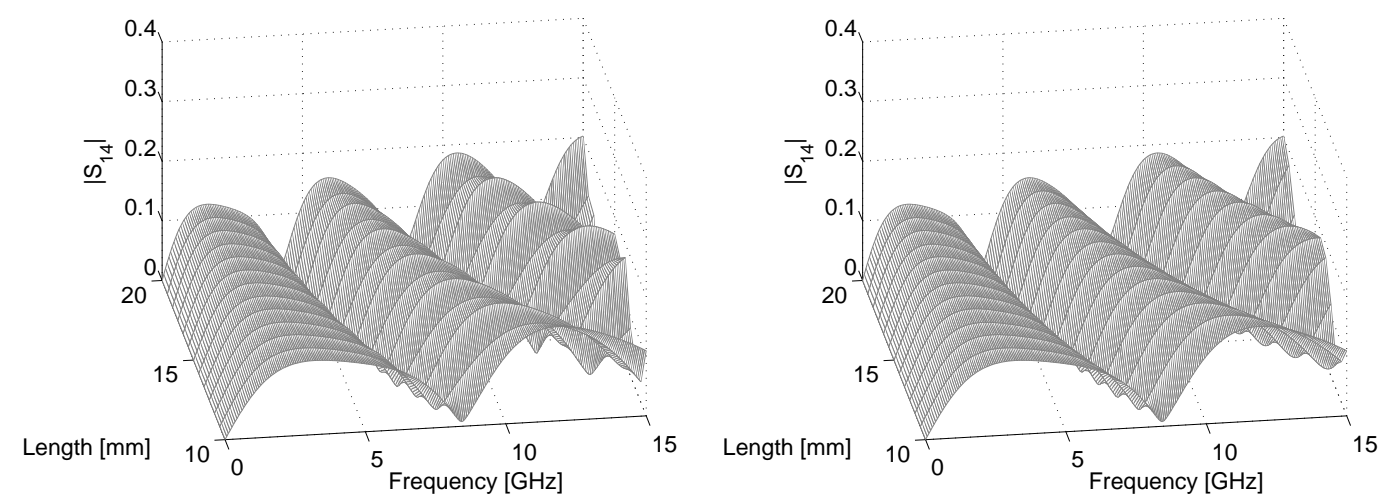

Figure 9. Magnitude of the trivariate macromodel of $\mathbf{S}_{14}(s, L, S)$ for $S=300 \mu \mathrm{m}$ by means of the old technique $[4,5]$ (left) and the new technique (right).
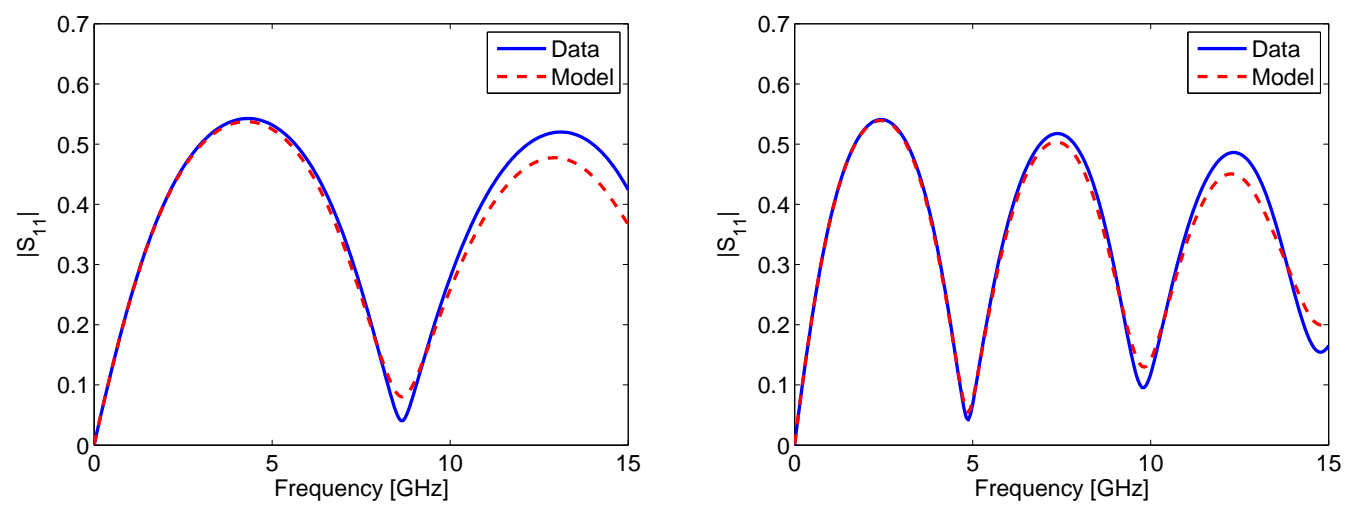

Figure 10. Magnitude of the trivariate macromodel of $\mathbf{S}_{11}(s, L, S)(L=\{10.6,19.4\} \mathrm{mm}, S=300 \mu \mathrm{m})$ by means of the old technique $[4,5]$.

\section{Conclusions}

We have presented a novel parametric macromodeling method for scattering representations.

The overall stability and passivity of the parametric macromodel are guaranteed, while poles and residues are parameterized indirectly. The proposed method is based on an efficient and 

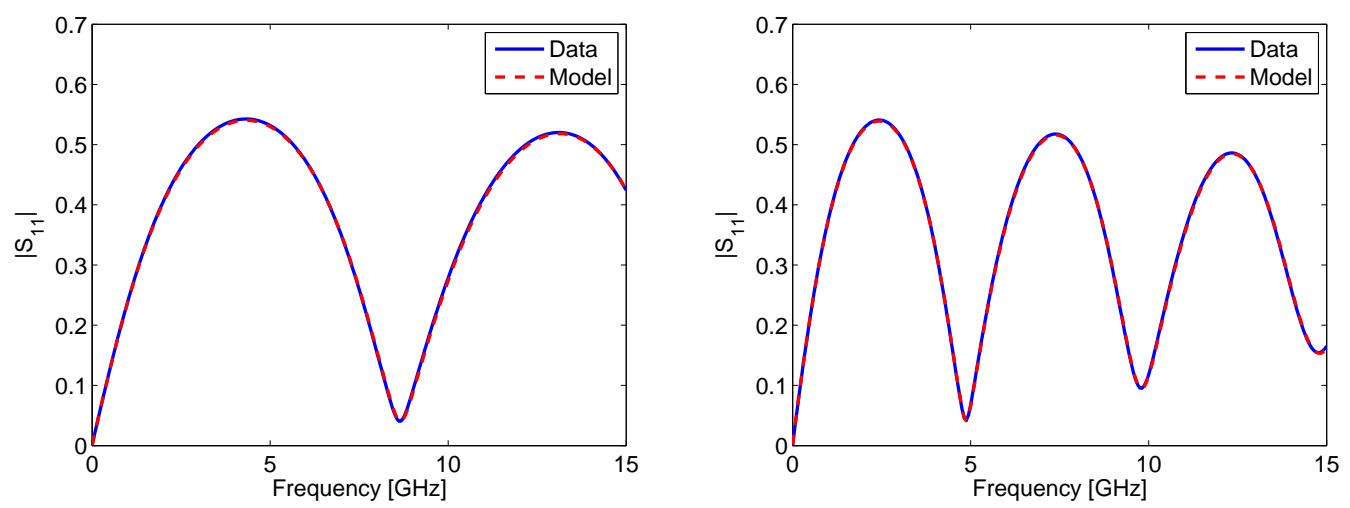

Figure 11. Magnitude of the trivariate macromodel of $\mathbf{S}_{11}(s, L, S)(L=\{10.6,19.4\} \mathrm{mm}, S=300 \mu \mathrm{m})$ by means of the new technique.
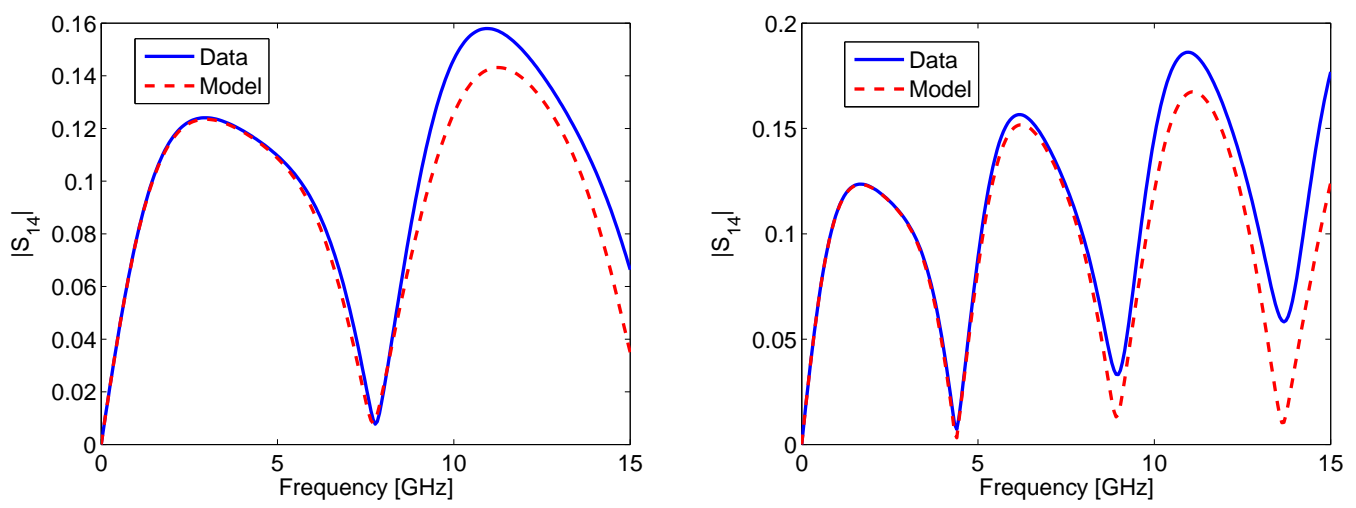

Figure 12. Magnitude of the trivariate macromodel of $\mathbf{S}_{14}(s, L, S)(L=\{10.6,19.4\} \mathrm{mm}, S=300 \mu \mathrm{m})$ by means of the old technique $[4,5]$.

reliable combination of rational identification, a procedure to find amplitude and frequency scaling system coefficients, and positive interpolation schemes. Numerical simulations confirm the capability of the proposed method of accurately describing dynamic electromagnetic systems, while preserving stability and passivity over the complete design space. 

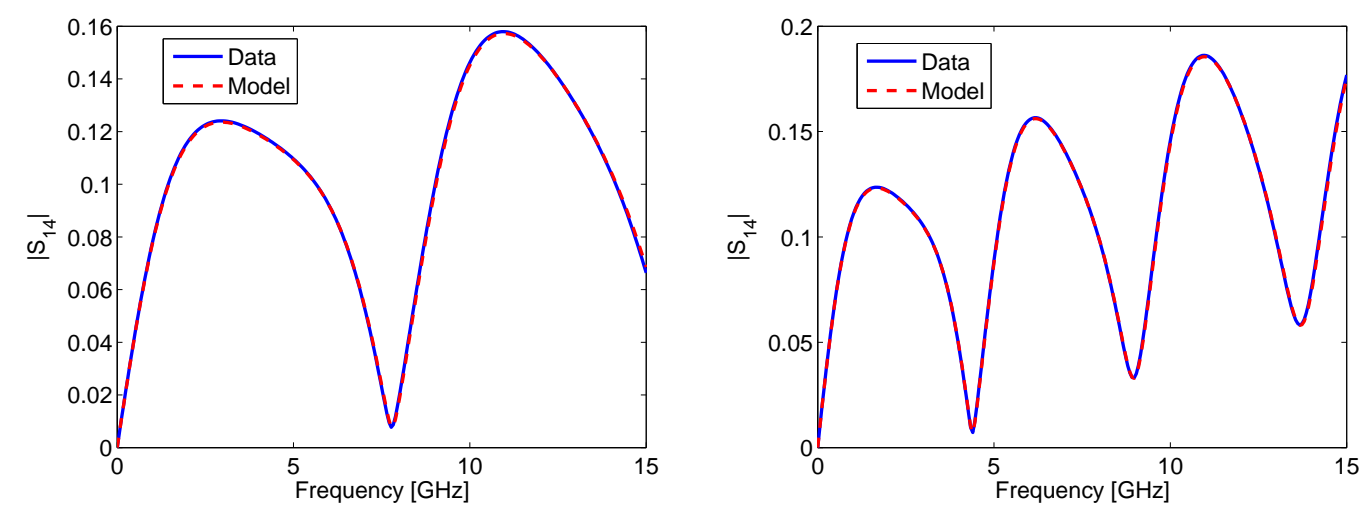

Figure 13. Magnitude of the trivariate macromodel of $\mathbf{S}_{14}(s, L, S)(L=\{10.6,19.4\} \mathrm{mm}, S=300 \mu \mathrm{m})$ by means of the new technique.

\section{REFERENCES}

1. D. Deschrijver, T. Dhaene and D. De Zutter. Robust parametric macromodeling using multivariate orthonormal vector fitting. IEEE Trans. Microw. Theory Tech., 56(7):1661-1667, July 2008.

2. P. Triverio, S. Grivet-Talocia, and M. S. Nakhla. A parameterized macromodeling strategy with uniform stability test. IEEE Trans. Adv. Packag., 32(1):205-215, February 2009.

3. F. Ferranti, L. Knockaert, and T. Dhaene. Guaranteed passive parameterized admittance-based macromodeling. IEEE Trans. Adv. Packag., 33(3):623 -629, August 2010.

4. F. Ferranti, L. Knockaert, and T. Dhaene. Parameterized S-parameter based macromodeling with guaranteed passivity. IEEE Microw. Wireless Compon. Lett., 19(10):608-610, October 2009.

5. F. Ferranti, L. Knockaert, and T. Dhaene. Passivity-preserving interpolation-based parameterized macromodeling of scattered S-data. IEEE Microw. Wireless Compon. Lett., 20(3):133-135, March 2010.

6. F. Ferranti, L. Knockaert, T. Dhaene, G. Antonini, and D. De Zutter. Parametric macromodeling for tabulated data based on internal passivity. IEEE Microw. Wireless Compon. Lett., 20(10):533 - 535, Oct. 2010.

7. F. Ferranti, L. Knockaert, T. Dhaene, and G. Antonini. Passivity-preserving parametric macromodeling for highly dynamic tabulated data based on Lur'e equations. IEEE Trans. Microw. Theory Tech., 58(12):3688 -3696 , Dec. 2010.

Copyright (c) 2007 John Wiley \& Sons, Ltd.

Int. J. Numer. Model. 2007; 01:1-6

Prepared using jnmauth.cls 
8. P. Triverio, M.S. Nakhla, and S. Grivet-Talocia. Passive parametric macromodeling from sampled frequency data. In IEEE 14th Workshop on Signal Propagation on Interconnects (SPI), 2010, pages $117-120$, May 2010.

9. J. De Caigny, J. F. Camino, and J. Swevers. Interpolating model identification for SISO linear parametervarying systems. Mechanical Systems and Signal Processing, 23(8):2395 - 2417, 2009.

10. P Gahinet, A. Nemirovski, A. J. Laub, and M. Chilali. LMI Control Toolbox User's Guide,Version 1. The Mathworks, Inc., 1995.

11. G. Balas, R. Chiang, A. Packard, and M. Safonov. Robust Control Toolbox User's Guide, Version 3. The Mathworks, Inc., 2005.

12. B. Gustavsen and A. Semlyen. Rational approximation of frequency domain responses by vector fitting. IEEE Trans. Power Delivery, 14(3):1052-1061, July 1999

13. D. Saraswat, R. Achar and M. Nakhla. On passivity enforcement for macromodels of S-parameter based tabulated subnetworks. In Proc. of the IEEE Int. Symp. on Circuits and Systems, volume 4, pages 3777-3780, May 2005

14. T. Dhaene, D. Deschrijver, and N. Stevens. Efficient algorithm for passivity enforcement of S-parameter based macromodels. IEEE Trans. Microw. Theory Tech., 57(2):415-420, February 2009.

15. G. Allasia. A class of interpolating positive linear operators: Theoretical and computational aspects. In S. P. Singh, editor, Recent Developments in Approximation Theory, Wavelets and Applications, pages 1-36, Dordrecht, 1995. Kluwer.

16. G. Allasia. Simultaneous interpolation and approximation by a class of multivariate positive operators. Numerical Algorithms, 34(2):147-158, December 2003.

17. W. A. Weiser and S. E. Zarantonello. A note on piecewise linear and multilinear table interpolation in many dimensions. Mathematics of Computation, 50(181):253-264, Jan. 1988.

18. H. W. Kuhn. Some combinatorial lemmas in topology. IBM Journal of Research and Development, $4(5): 518-524,1960$.

19. D. F. Watson. Computing the n-dimensional Delaunay tessellation with application to Voronoi polytopes. The Computer Journal, 24(2):167-172, Febr. 1981.

20. C. Bradford Barber, David P. Dobkin, and Hannu Huhdanpaa. The quickhull algorithm for convex hulls. ACM Trans. Math. Softw., 22(4):469-483, 1996.

21. R. Rohrer, H. Nosrati. Passivity considerations in stability studies of numerical integration algorithms. IEEE Transactions on Circuits and Systems, 28(9):857-866, Sep. 1981. 
22. L. W. Nagel. SPICE: A computer program to simulate semiconductor circuits. Electr. Res. Lab. Report ERL M520, University of California, Berkeley, May 1975.

23. E. A. Guillemin. Synthesis of Passive Networks. John Wiley and Sons, New York, 1957.

24. S. Boyd, L. El Ghaoui, E. Feron and V. Balakrishnan. Linear Matrix Inequalities in System and Control Theory, volume 15. SIAM, Philadelphia, PA, 1994.

25. B. D. Anderson, S. Vongpanitlerd. Network Analysis and Synthesis. Prentice-Hall, Englewood Cliffs, NJ, 1973.

26. E. W. Cheney. Multivariate approximation theory: Selected topics. In $C B M S$, volume 51, Philadelphia, PA, 1986. SIAM.

27. A. E. Ruehli. Equivalent circuit models for three dimensional multiconductor systems. IEEE Trans. Microw. Theory Tech., 22(3):216-221, March 1974.

28. G. Antonini, A.E. Ruehli, and Chuanyi Yang. PEEC modeling of dispersive and lossy dielectrics. IEEE Trans. Adv. Packag., 31(4):768 -782, Nov. 2008

29. A.R.Djordjević, R.M. Biljić, V.D. Likar-Smiljanić, T.K. Sarkar. Wideband frequency-domain characterization of FR-4 and time-domain causality. IEEE Transactions on Electromagnetic Compatibility, 43(4):662-667, Nov. 2001.

30. Simberian Inc., Seattle. Simbeor, Electromagnetic Simulation Environment with 3D Full-Wave Field Solver for Multi-Layered Circuits.

31. C. R. Paul. Analysis of Multiconductor Transmission Lines. John Wiley \& Sons, New York, NY, second edition, 2008.

\section{AUTHORS' BIOGRAPHIES}

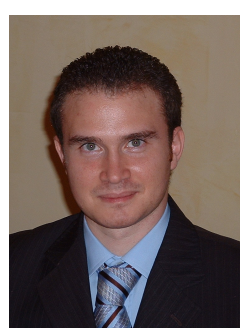

Francesco Ferranti received the B.S. degree (summa cum laude) in electronic engineering from the Università degli Studi di Palermo, Palermo, Italy, in 2005 and the M.S. degree (summa cum laude and honors) in electronic engineering from the Università degli Studi dell'Aquila, Copyright @ 2007 John Wiley \& Sons, Ltd. Int. J. Numer. Model. 2007; 01:1-6 Prepared using jnmauth.cls 
L'Aquila, Italy, in 2007. He is currently working towards the Ph.D. degree in the Department of Information Technology (INTEC), Ghent University, Ghent, Belgium. His research interests include parametric macromodeling, parameterized model order reduction, EMC numerical modeling, system identification.

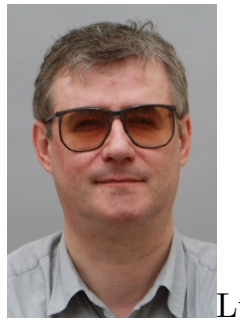

Sc. Degree in telecommunications engineering and the $\mathrm{Ph}$. D. Degree in electrical engineering from Ghent University, Belgium, in 1974, 1977 and 1987, respectively. From 1979 to 1984 and from 1988 to 1995 he was working in North-South cooperation and development projects at the Universities of the Democratic Republic of the Congo and Burundi. He is presently affiliated with the Interdisciplinary Institute for BroadBand Technologies (www.ibbt.be) and a professor at the Dept. of Information Technology, Ghent University (www.intec.ugent.be). His current interests are the application of linear algebra and adaptive methods in signal estimation, model order reduction and computational electromagnetics. As author or co-author he has contributed to more than 100 international journal and conference publications. He is a member of MAA, SIAM and a senior member of IEEE.

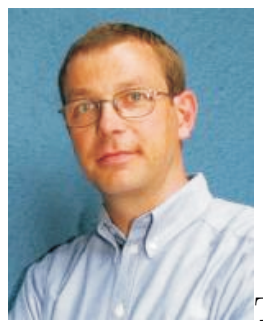

Tom Dhaene was born in Deinze, Belgium, on June 25, 1966. He received the Ph.D. degree in electrotechnical engineering from the University of Ghent, Ghent, Copyright (c) 2007 John Wiley \& Sons, Ltd. Int. J. Numer. Model. 2007; 01:1-6 Prepared using jnmauth.cls 
Belgium, in 1993. From 1989 to 1993, he was Research Assistant at the University of Ghent, in the Department of Information Technology, where his research focused on different aspects of full-wave electro-magnetic circuit modeling, transient simulation, and time-domain characterization of high-frequency and high-speed interconnections. In 1993, he joined the EDA company Alphabit (now part of Agilent). He was one of the key developers of the planar EM simulator ADS Momentum. Since September 2000, he has been a Professor in the Department of Mathematics and Computer Science at the University of Antwerp, Antwerp, Belgium. Since October 2007, he is a Full Professor in the Department of Information Technology (INTEC) at Ghent University, Ghent, Belgium. As author or co-author, he has contributed to more than 150 peer-reviewed papers and abstracts in international conference proceedings, journals and books. He is the holder of 3 US patents.

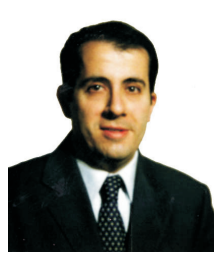

Giulio Antonini received his Laurea degree (summa cum laude) in Electrical Engineering in 1994 from the Università degli Studi dell'Aquila and the Ph.D. degree in Electrical Engineering in 1998 from University of Rome "La Sapienza". Since 1998 he has been with the UAq EMC Laboratory, Department of Electrical Engineering of the University of L'Aquila where he is currently Associate Professor. His research interests focus on EMC analysis, numerical modeling and in the field of signal integrity for highspeed digital systems. He has authored or co-authored more than 170 technical papers and 2 book chapters. Furthermore, he has given keynote lectures and chaired several special sessions at international conferences. He has been the recipient of the IEEE Transactions 
on Electromagnetic Compatibility Best Paper Award in 1997, the CST University Publication Award in 2004, the IBM Shared University Research Award in 2004, 2005 and 2006, the IETSMT Best Paper Award in 2008. In 2006 he has received a Technical Achievement Award from the IEEE EMC Society "for innovative contributions to computational electromagnetic on the Partial Element Equivalent Circuit (PEEC) technique for EMC applications". He holds one European Patent. 